\title{
Modelo de Responsabilidad Social Empresarial en la PYME, caso Tula-Tepeji, México
}

\author{
Model of Corporate Social Responsibility in SMEs, case Tula-Tepeji, Mexico. \\ Ismael Acevedo Sánchez ${ }^{a}$, Alfredo Castillo Trejo ${ }^{b}$ y Ma. de Lourdes Ortega Montiel ${ }^{c}$
}

\begin{abstract}
:
Corporate Social Responsibility (CSR) has become a practice that is not exclusive to large organizations, small and medium-sized enterprises (SMEs) must to adapt strategies to achieve extra benefits in the sector in which they develop, that's how it comes, the Ibero-American Network of Corporate Social Responsibility (RIRSEP), whose purpose is to establish a CSR model for Ibero-American SMEs, the aim of this work is; to integrate a CSR model for the case of SMEs in the Tula-Tepeji region in the Hidalgo State. The method used consisted of an exploratory study, the data collection was by a survey composed of 77 items. Among the main findings were identified that companies in the region develop scattered social responsibility activities, but are open to input and suggestions that can be provided, finally the research was obtained a model of CSR it will be presented as a contribution of the Tula node to the RIRSEP for its evaluation and integration in the model that the network has proposed to contribute to Ibero-American SMEs.
\end{abstract}

\section{Keywords:}

Corporate Social Responsibility, CSR Model, Tula-Tepeji Region, RIRSEP

\section{Resumen:}

La responsabilidad Social Empresarial (RSE) se ha convertido en una práctica que no es exclusiva de las grandes organizaciones, las pequeñas medianas empresas (PYME) tienen la obligación de adaptar estrategias para lograr beneficios extras en el sector en el que se desenvuelvan, sí deciden impactar el mercado en el que se encuentren, es así como surge la la Red Iberoamericana de Responsabilidad Social Empresarial (RIRSEP) cuyo propósito es establecer un modelo de RSE para las PYME de Iberoamérica, el objetivo de este trabajo es; integrar un modelo de RSE para el caso de las PYME de la región TulaTepeji en el Estado de Hidalgo. El método consistió en un estudio exploratorio, la recolección de información fue por medio de un instrumentó integrado por 77 reactivos. Dentro de los principales hallazgos se identificó que las empresas de la región desarrollan escazas actividades de RSE, pero están abiertas a las aportaciones y sugerencias que en materia se les pueda brindar, finalmente de la investigación se obtuvo un modelo de RSE mismo que se presentará como aportación del nodo Tula a la RIRSEP para su evaluación e integración en el modelo que la red se ha propuesto aportar a las PYME de Iberoamérica.

\section{Palabras Clave:}

Modelo de RSE, Región Tula-Tepeji, Responsabilidad Social Empresarial, RIRSEP

\footnotetext{
a Autor de Correspondencia, Universidad Tecnológica de Tula-Tepeji, ORCID: 0000-0002-4167-3047, Email: Ismael.acevedo@uttt.edu.mx b Universidad Tecnológica de Tula-Tepeji, ORCID: 0000-0002-1063-497, Email: alfredo.castillo@uttt.edu.mx

c Universidad Tecnológica de Tula-Tepeji, ORCID: 0000-0003-0005-1935, Email: mariadelourdes.ortega@uttt.edu.mx
} 


\section{Introducción}

Las pequeñas y medianas empresas (PYME) han sido sujeto de innumerables estudios, no solo por su contribución al empleo, sino por su importancia en cuanto a su aportación al producto interno bruto. De acuerdo con datos del INEGI (2014), en México existen registradas alrededor de 5.1 millones de unidades económicas, de las cuales 4 . 897,141 son micro; 221,195 son pequeñas; 15,432 medianas, en contraste con las 9,788 grandes. Como se puede observar en México, las PYME constituyen $99.8 \%$ del total del universo empresarial. Por otra parte, de acuerdo con Vanderberg citado por Saavedra (2014), comenta que la importancia de las PYMES también radica en que las pequeñas empresas pueden ayudar a reducir la pobreza en los países, puesto que generan gran cantidad de empleo, con niveles adecuados de calidad y bajo costo de bienes y servicios.

Por lo tanto para las PYME, es necesario mejorar el entorno económico y el apoyo directo a las empresas con instrumentos que generen las condiciones que contribuyan a su establecimiento, crecimiento y consolidación; no obstante muchas PYME no logran desarrollarse o consolidarse, su tasa de éxito se encuentra por debajo de la media mundial con valores alrededor de los 25 y $30 \%$, contra un $40 \%$ que representa esta media; se han identificado una serie de elementos por los cuales estas empresas suelen fracasar, dentro de los cuales los de mayor influencia son; financiamiento, falta de asesoría empresarial, deficiente administración, recursos humanos no calificados, desconocimiento de mercados, desconocimiento de la tecnología e innovación, además de una deficiente organización; todos estos factores contribuyen a una falta de competitividad de las PYME en nuestro país. Es necesario apoyar a estas entidades económicas a superar los diversos obstáculos con los que se enfrentan para su crecimiento y competitividad; como la falta de una estructura productiva, el acceso a los créditos, la falta de una cultura de conocimiento, la poca innovación hacia los clientes y proveedores, el acceso y utilización a la tecnología, además de la falta de certificaciones de calidad, o de responsabilidad social empresarial (RSE).

Con base en el conocimiento general de lo que es la PYME revisado anteriormente, sus características y situación actual, así como sus necesidades y oportunidades de crecimiento, es necesario indagar una herramienta estratégica para el buen desarrollo y estabilidad de ésta, la cual sin duda podría ser, el sello de la Responsabilidad Social Empresarial (RSE).

De acuerdo con Espinosa, R. Contreras, R. y Rodríguez R. (2016), debido a la situación que estamos viviendo día con día, una época más globalizada en todos los sentidos, y en la cual solo 
los más aptos podrán sobrevivir a las demandas de un mercado tan cambiante y competitivo, es necesario que las PYME mexicanas se adapten de la mejor manera posible a estos cambios, para ello es indispensable implementar ciertos criterios o reglas que guíen su labor; la respuesta al anterior planteamiento podría ser la obtención del distintivo que puede ayudar a las PYME mexicanas a lograr sus objetivos, el cual tienen implantado las empresas socialmente responsables (ESR), ya que este tipo de organizaciones tienen un sistema muy bien definido, en el cual sobresalen y por ende llegan a tener éxito, esto lo podemos ver claramente en nuestro país, puesto que hay muchas entidades que llegan a triunfar debido a que aplican dicho distintivo (Alto nivel 2009).

Con relación a lo anterior Villafan, K. y Ayala, D. (2014), señalan que, dentro de la RSE se generan beneficios al interior de la empresa, tales como una mejor calidad del entorno laboral, relaciones más cordiales y sólidas con los grupos de interés, entre otros; asimismo, beneficios al exterior de ella mediante una mayor vinculación con la comunidad y un mayor involucramiento con temas medioambientales. Estos beneficios pueden ser traducidos como ventajas competitivas sobre el resto de los competidores.

Las empresas comprometidas con la responsabilidad social experimentan importantes beneficios cualitativos y cuantitativos, tales como el desempeño financiero positivo, la reducción de costos operativos, la mejora de su imagen, reputación, lealtad de sus clientes y empleados e incremento en sus ventas, entre otros. Cada vez más, las empresas mexicanas y las transnacionales que operan en nuestro territorio reconocen que no es posible separar la participación corporativa de la responsabilidad social, pues empieza a ser notorio que las empresas más admiradas están relacionadas con indicadores de inversión social empresarial. Ejemplo de ello es que entre las iniciativas que buscan reconocer las acciones que realizan las empresas con beneficio de la comunidad y en beneficio de ella se destacan varias organizaciones como; la actividad del Centro Mexicano para la Filantropía (CEMEFI), la Unión Social de Empresarios de México (USEM), el Desarrollo Empresarial Mexicano (DESEM), la Confederación Patronal de la República Mexicana (COPARMEX) y la Revista Expansión. Conjuntamente se han dado a la labor de premiar anualmente, a partir del 2000, a Las Mejores Prácticas de Responsabilidad Social Empresarial, lo cual constituye el primer reconocimiento mundial a las prácticas (calidad de vida, medio ambiente y vinculación con la comunidad) y no a las empresas (Mercado, P. y García, P. 2007).

No obstante, lo anterior la presente investigación plantea el siguiente problema: "Actualmente no se cuenta con un modelo de RSE establecido en las empresas de la región Tula Tepeji, en el Estado de Hidalgo, México, con el cual puedan basarse para 
adoptar este sistema y mejorar su respuesta a sus grupos de interés", el establecer un modelo permitirá a las empresas de la región contar con una directriz específica que les permita iniciar las gestiones para obtener un distintivo de ESR. Por lo tanto, para el presente trabajo se ha establecido como objetivo de investigación: Integrar un modelo de RSE para las PYME de la región TulaTepeji en el Estado de Hidalgo México, a partir del análisis de las empresas socias del nodo Tula, que permita identificar su situación actual en cuanto a RSE de las organizaciones en el área sujeto de estudio.

Este trabajo es una investigación no experimental de tipo exploratoria por medio del instrumento adaptado e integrado por siete diferentes sesiones con 77 reactivos. Se trata de una muestra no probabilística de 5 empresas, correspondientes al nodo Tula. Si bien esto pudiera representar en esencia una limitante al desarrollo del producto, el presente trabajo forma parte del estudio realizado por la Red Iberoamericana de Responsabilidad Social Empresarial (RIRSEP), al cual pertenece el nodo Tula, y del cual se desprende una investigación de tipo longitudinal cuantitativa; el alcance de esta investigación radica en que presentará una propuesta de modelo de RSE a la RIRSEP para obtener un modelo integrado que se pueda utilizar para las empresas de Iberoamérica. De tal manera que las aportaciones de cada nodo que integran la Red justifica de manera significativa el estudio que comenzó a desarrollarse a partir de febrero del 2016.

\section{DESARROLLO}

La Responsabilidad Social Empresarial (RSE) tiene sus orígenes en 1953 con la publicación Social Responsibility of the Businessman del economista norteamericano Howard R. Bowen. Desde sus inicios hasta el día de hoy han surgido una gran diversidad de enfoques teóricos entre los que se encuentran el moralista, contractual y utilitarista, o el instrumental, político, integrador y ético (Garriga y Melé, 2004).

El presente trabajo no pretende ser un ser un estudio analítico de la evolución de la RSE, pero es importante abordar sus principales conceptos en función de sustentar las bases que se estarán trabajando a lo largo de este primer acercamiento de la investigación exploratoria, además de cimentar las bases para el desarrollo de la investigación longitudinal que se desarrollará con los demás miembros de la RIRSEP.

En ese sentido, y de acuerdo con García y Zabala (2008: p115), mencionan que La Responsabilidad Social Empresarial (RSE) es "un instrumento corporativo que implica un compromiso de las em presas a través de la aplicación sistemática de recursos para respetar y promover los derechos de las personas, el crecimiento de la sociedad y el cuidado

del ambiente". 
Por otra parte, Marín (2007, p. 245), resalta que "la importancia de utilizar teorías de identidad social, significa hasta qué punto las personas se identifican con la empresa"; sin embargo, la RSE puede definirse como un contrato social. Al respecto la Secretaria de Economía en México señala que, la responsabilidad social empresarial, se define como la contribución activa y voluntaria al mejoramiento social, económico y ambiental por parte de las empresas, con el objetivo de mejorar su situación competitiva, valorativa y su valor añadido.

Así, pues, la responsabilidad que tienen las empresas ante los impactos de sus decisiones y actividades que ocasionan en la sociedad y el medio ambiente deben ser expresadas en un comportamiento organizacional que tome en consideración las expectativas de sus partes interesadas o stakeholders, que contribuya al desarrollo sostenible, la salud y el bienestar de la sociedad, que cumpla con la legislación vigente y sobre todo que esté integrada en toda la organización y forme parte de su identidad, cultura y valores (Moreno, Z. y Graterol, D. 2001).

Por otra parte, encontramos que el reciente interés de organismos internacionales, de carácter público y privado, por este tema ha influido en gran medida a que éstos participen activamente en la homogenización del concepto. En particular, la Organización Internacional para la Estandarización comenzó a trabajar desde el año
2005 en la elaboración de la norma ISO 26000 sobre RSE y ha reunido desde entonces a más de 450 expertos y 210 observadores de 99 países miembros y 42 organizaciones (ISO, 2010). Esta organización define la RSE como: "la responsabilidad de una organización ante los impactos que sus decisiones y actividades ocasionan en la sociedad y el medio ambiente mediante un comportamiento ético y transparente que contribuya al desarrollo sostenible, incluyendo la salud y el bienestar de la sociedad, que toma en consideración las expectativas de sus partes interesadas, que cumpla con la legislación aplicable y sea coherente con la normativa internacional de comportamiento, y que está integrada en toda la organización y se lleva a la práctica en sus relaciones “(ISO, 2010).

De acuerdo con la norma ISO 26000, es la responsabilidad de una organización ante los impactos que sus decisiones y actividades que ocasionan en la sociedad y el medio ambiente, mediante un comportamiento ético y transparente que:

Contribuya al desarrollo sostenible, incluyendo la salud y el bienestar de la sociedad.

Tome en consideración las expectativas de sus partes interesadas.

Cumpla con la legislación aplicable y sea coherente con la normativa internacional de comportamiento. 
Esté integrada en toda la organización y se lleve a la práctica en sus relaciones.

La RSE es considerada parte fundamental de una nueva cultura de reconocimiento y exigencia mutua entre las empresas, la sociedad y las instituciones, la RSE es un marco de relación que comprende a todos los sectores; administración, empresas, organizaciones y ciudadanía, por lo tanto, la aplicación en las Pequeñas y Medianas Empresas (PYME) es más natural, dada su mayor relación con el entorno y su proximidad a los clientes. De hecho, las PYME vienen aplicando prácticas de RSE de forma habitual y ligada a su quehacer diario, aún sin conocer el concepto y sin llamarlas por ese nombre. Y un dato crucial: sin la implicación de las PYME, la RSE no puede tener un impacto real en la sociedad.

De acuerdo Rivero (2006), La Responsabilidad Social presenta un menor grado de desarrollo en las Pequeñas y Medianas Empresas (PYME) que en las grandes compañías, por dos razones fundamentales: en primer lugar porque en el entorno de las PYME existe una menor necesidad en la asunción del concepto, dado que estas compañías no han vivido la pérdida de confianza que han sufrido las grandes empresas y en segundo lugar, porque las PYME tienen que enfrentarse a ciertas barreras de entrada que dificultan su implementación: falta de conocimiento, carencia de personal preparado, limitación de recursos en una inversión cuyo retorno se produce en el medio largo plazo. No obstante, esta diferenciación inicial no debe inducir al error de pensar que las políticas de Responsabilidad Social de las PYME, sean distintas de las que siguen las grandes empresas, puesto que se asienta en los tres pilares básicos de la responsabilidad social y en los mismos principios y actividades que aplican estas compañías, existiendo más diferencias cuantitativas (menores recursos) que cualitativas. Además, las PYME cuentan con ciertas ventajas: mayor flexibilidad, especialización, y mayor capacidad relacional y de adaptación a las necesidades de un entorno cambiante y a las de sus interlocutores sociales.

Según Páez (2007, p. 26), en la PYME no se cuenta con una estrategia formalmente definida de RSE; asimismo no se contemple a una unidad orgánica, ni tampoco una persona responsable, que impulse la planificación de la RSE como componente gerencial; lo cual, conlleva a que los planes en relación con el desarrollo de alguna actividad relacionada con RSE se lleve a efecto sin la debida planificación en concordancia con los objetivos de rentabilidad de las PYME y su visión estratégica de largo plazo.

En este orden de ideas, Páez (2007) y Moreno (2006), consideran importante establecer evaluaciones que permitan determinar el impacto de los programas de RSE debido a que no se tienen establecidos sistemas de control e indicadores que permitan evaluar los cambios en relación con la 
eficiencia de dichos programas o acciones. También reconocen la existencia de personal profesional de alto nivel, lo cual ha sido considerado una fortaleza para el establecimiento de la RSE como una estrategia gerencial para impulsar mejoras en los niveles competitivos de las empresas.

En México no existe mucha información relacionada con datos estadísticos y mejores prácticas que permitan a las empresas tomar decisiones estratégicas en torno a la gestión de la responsabilidad social empresarial, con base en esta situación la Universidad Tecnológica de TulaTepeji (UTTT), por medio de su cuerpo académico de Polos de Desarrollo Económico, perteneciente al programa educativo de Desarrollo de Negocios, aceptó la invitación para participar en; la Red Iberoamericana de Responsabilidad Social Empresarial, que se crea para impulsar la incorporación de criterios de Responsabilidad Social Empresarial a la gestión de las Pequeñas y Medianas Empresas con el propósito de contribuir a mejorar su competitividad en un marco de actuación socialmente responsable, por medio de una investigación longitudinal en un periodo de cuatro años. El equipo de trabajo está integrado por un grupo de investigadores la Universidad Gerardo Barrios (El Salvador), Universidad federal de Santa Catarina (Brasil), Universidad Simón Bolívar (Colombia), Universidad de Quindío (Colombia), Universidad ORT (Uruguay), Pontificia Universidad Católica Madre y Maestra (República Dominicana), Universidad de Alicante (España), Universidad de Barcelona (España), Universidad Tecnológica de TulaTepeji (México), Universidad Autónoma de Ciudad Juárez (México) y la Universidad Nacional del Litoral (Argentina); y por la organización empresarial: Asociación Colombiana de la Micro, Pequeña y Mediana Empresa -ACOPI-, todos coordinados por la Universidad Gerardo Barrios, se pretende que este accionar conjunto genere un círculo virtuoso que comience con el compromiso de las PYME en asumir y/o profundizar la utilización de prácticas de RSE que les permita fortalecer su imagen y reputación y las lleve a obtener una ventaja competitiva generando valor económico, social y ambiental que contribuya al desarrollo sustentable del territorio.

Para el desarrollo de la investigación cada institución participante, se identificó como "Nodo", la Universidad Tecnológica de TulaTepeji se constituye como uno de los dos nodos que participan de México, junto con la Universidad Autónoma de Ciudad Juárez, para el caso particular de la UTTT, se contó con la valiosa colaboración de 5 organizaciones, quienes amablemente han destinado parte de su tiempo para las labores propias del estudio y que en lo sucesivo se denominan; "empresas socias", estas empresas son:

1. Arlequín Abarrotero S. de R.L. de C.V. 
2. Centro Especializado en Enfermedades de La Piel.

3. Colegio "Erasmo de Rotterdam", Preescolar y Primaria.

4. Jubileo Azul, S.A. de C.V.

5. Prometrica S.A de C.V.

Para la primera parte de la investigación se ha establecido realizar un diagnóstico de las PYME socias de cada nodo y con los resultados se establecerá un modelo de RSE para PYME en Iberoamérica.

Los resultados presentados en este estudio corresponden específicamente a los obtenidos en la primera parte de la investigación para caso del nodo Tula-Tepeji, en el estado de Hidalgo, México, mismos que servirán para integrar los trabajos de la Red Iberoamericana de Responsabilidad Social Empresarial que coordina la Universidad Gerardo Barrios de El Salvador.

\section{MÉTODO}

La presente investigación será una investigación no experimental debido a que se desarrolla en el campo de las ciencias administrativas aplicado a las PYME de la región Tula-Tepeji, el método a utilizar será analítico-sintético, de acuerdo con Bernal (2010), este tipo de estudios analiza los hechos partiendo de la descomposición del objeto de estudio en cada una de sus partes para estudiarlas en forma individual, y luego se integran esas partes para estudiarlas de manera holística; esta investigación también será transversal, en el sentido que se estudiará, las actividades que con relación a la RSE desarrollan la empresas de la región Tula-Tepeji, en un periodo especifico (Münch, 2012), en este caso comprendió de febrero de 2016 a junio de 2017. Para la recolección de información se aplicó un cuestionario adaptado e integrado por siete diferentes sesiones, en la primera sección se encuentra el área de "gobernabilidad", posteriormente "publico interno", seguido de "medio ambiente", "proveedores", "mercado", “comunidades" y "políticas públicas". Estas variables describen de manera general los elementos que deben de integrar las empresas que trabajen bajo un esquema de RSE. Este instrumento estuvo formado por 77 reactivos mixtos cuyas respuestas están limitadas a las posibles alternativas que el entrevistado percibe con relación a la RSE.

\section{POBLACIÓN Y MUESTRA}

El cuestionario fue aplicado por los integrantes del nodo Tula y contestado por directivos y/o dueños de 5 pequeñas y medianas empresas de la región Tula-Tepeji, en el Estado de Hidalgo (México). Se trata de una muestra no probabilística en cuanto a la elección de las empresas participantes, debido a que una de las mayores dificultades para este tipo de estudios se refiere a lograr el acceso a ellas, ya que sus directivos y/o propietarios son muy reacios a la investigación sobre temas relativos y similares a aspectos directivos y del factor humano y organizacional. De las empresas participantes las cinco empresas son PYME (debido a que el 
alcance del estudio está centrado en el análisis de las mismas) el $20 \%$ son del giro industrial y el $80 \%$ de servicios, dos tienen su razón social en el municipio de Tepeji del Río y los tres restantes en el de Tula de Allende.

\section{RESULTADOS}

De los datos obtenidos, se presenta a continuación una serie de graficas con un resumen general de las principales incidencias de las variables estudiadas, el análisis posterior de estas variables, servirán de base para realizar la propuesta de un modelo de RSE, que se adapte a las características de las PYME de la región Tula-Tepeji, en el estado de Hidalgo, México.

\section{Gráfica 1. Gobernabilidad}

\section{Gobernabilidad}

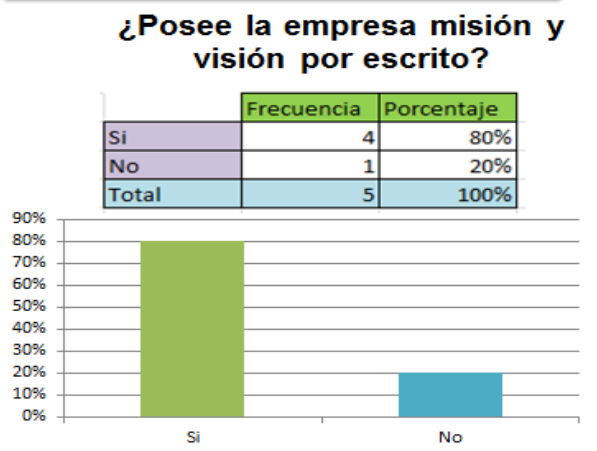

Fuente: Elaboración Propia

Gráfica 2. Procesos de RSE

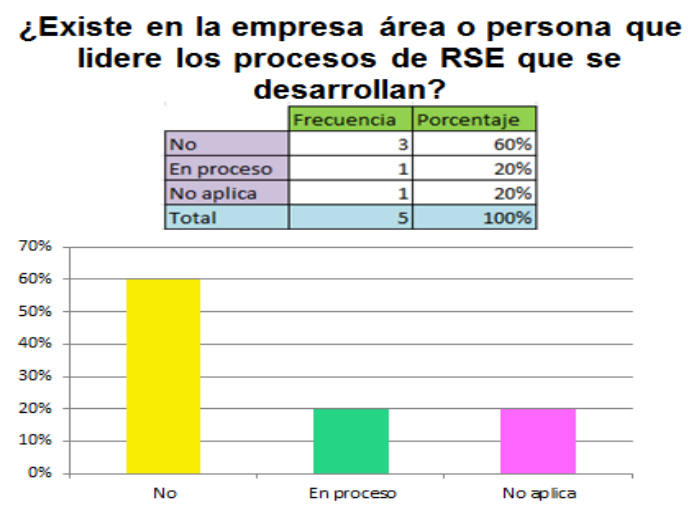

Gráfica 3. Uso de las TIC’S

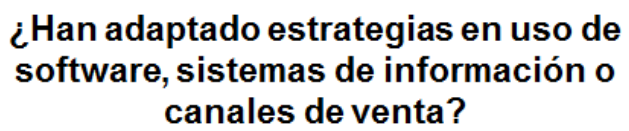

\begin{tabular}{|l|r|r|}
\cline { 2 - 3 } \multicolumn{1}{c|}{} & Frecuencia & Porcentaje \\
\hline Si & 2 & $40 \%$ \\
\hline No & 3 & $60 \%$ \\
\hline Total & 5 & $100 \%$ \\
\hline
\end{tabular}

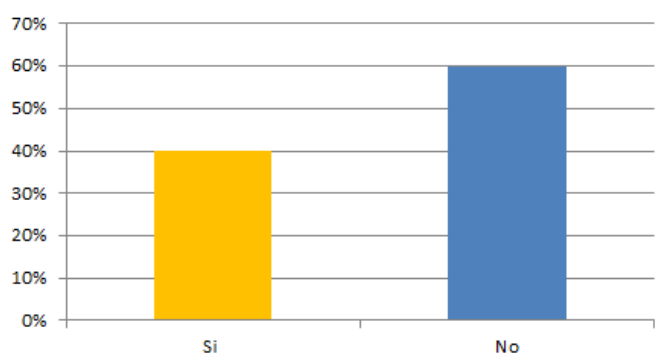

Fuente: Elaboración Propia

Gráfica 4. Público Interno

¿Establece la empresa contratos de trabajo por escrito con sus colaboradores y colaboradoras?

\begin{tabular}{|l|r|r|}
\cline { 2 - 3 } \multicolumn{1}{c|}{} & Frecuencia & Porcentaje \\
\hline No & 3 & $60 \%$ \\
\hline En proceso & 1 & $20 \%$ \\
\hline Total & 1 & $20 \%$ \\
\hline
\end{tabular}

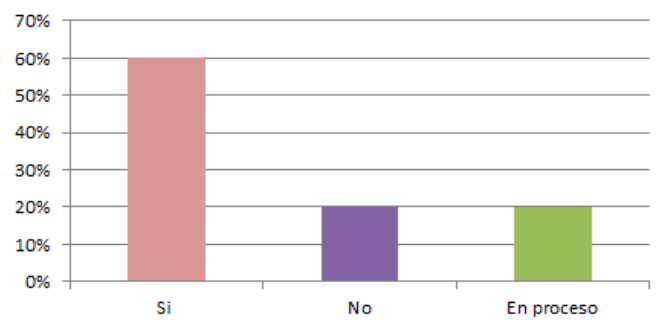

Fuente: Elaboración Propia

Gráfica 5. Normatividad

¿Cuenta su empresa con reglamento interno de trabajo?

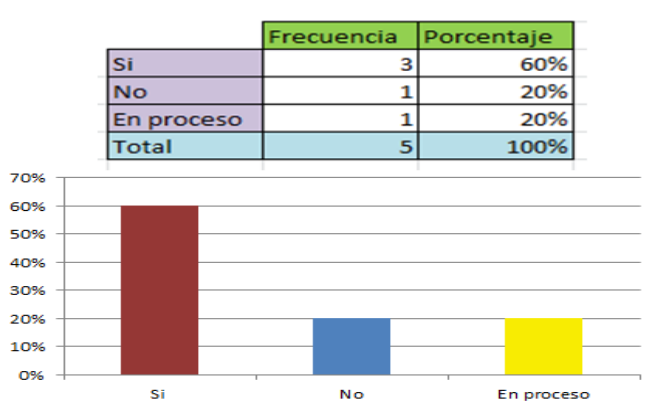


Gráfica 6. Programa de Inducción

¿Existe un programa formal de inducción para los nuevos colaboradores y colaboradoras?

\begin{tabular}{|l|r|r|}
\cline { 2 - 3 } \multicolumn{1}{c|}{} & Frecuencia & Porcentaje \\
\hline Si & 1 & $20 \%$ \\
\hline No & 3 & $60 \%$ \\
\hline En proceso & 1 & $20 \%$ \\
\hline Total & 5 & $100 \%$ \\
\hline
\end{tabular}

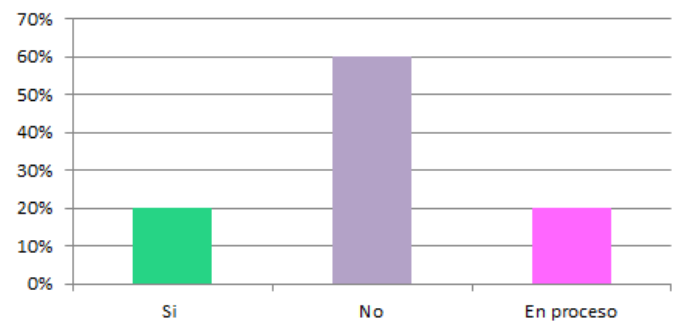

Fuente: Elaboración Propia

Gráfica 7. Reclutamiento y Selección

¿Cuenta la empresa con un procedimiento formal de reclutamiento y selección del personal?

\begin{tabular}{|l|r|r|}
\cline { 2 - 3 } \multicolumn{1}{c|}{} & Frecuencia & Porcentaje \\
\hline Si & 2 & $40 \%$ \\
\hline No & 1 & $20 \%$ \\
\hline En proceso & 2 & $40 \%$ \\
\hline Total & 5 & $100 \%$ \\
\hline
\end{tabular}

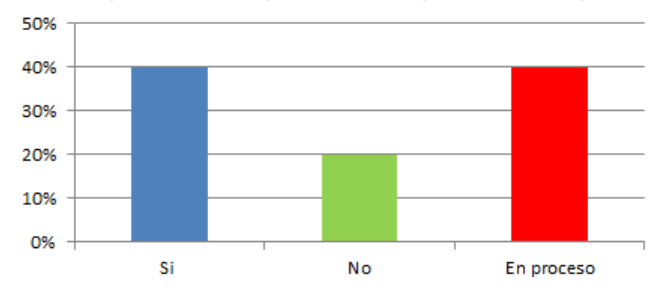

Fuente: Elaboración Propia

Gráfica 8. Desarrollo Profesional

¿Existen programas de desarrollo personaly profesional para las y los colabores?

\begin{tabular}{|l|r|r|}
\cline { 2 - 3 } \multicolumn{1}{c|}{} & Frecuencia & Porcentaje \\
\hline Si & 1 & $20 \%$ \\
\hline En proceso & 4 & $80 \%$ \\
\hline Total & 5 & $100 \%$ \\
\hline
\end{tabular}

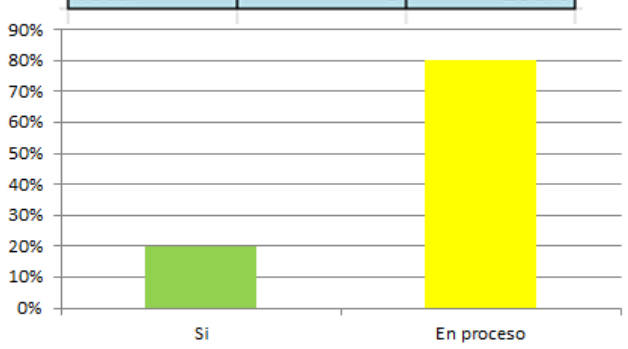

Gráfica 9. Medio Ambiente

\section{Medio ambiente}

\section{¿Se conocen todas las leyes ambientales nacionales que aplican a su negocio?}

\begin{tabular}{|l|r|r|}
\cline { 2 - 3 } \multicolumn{1}{c|}{} & Frecuencia & Porcentaje \\
\hline Si & 3 & $60 \%$ \\
\hline No & 2 & $40 \%$ \\
\hline Total & 5 & $100 \%$ \\
\hline
\end{tabular}

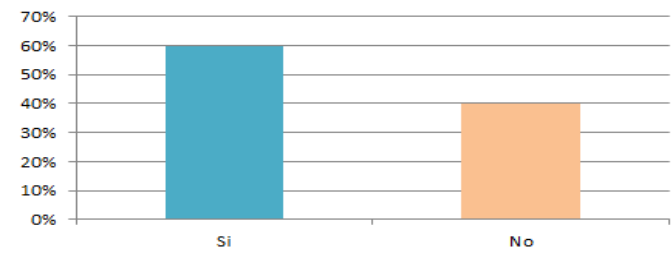

Fuente: Elaboración Propia

Gráfica 10. Leyes Ambientales

\section{¿Cumple con todas las leyes ambientales nacionales?}

\begin{tabular}{|l|r|r|}
\cline { 2 - 3 } \multicolumn{1}{c|}{} & Frecuencia & Porcentaje \\
\hline Si & 4 & $80 \%$ \\
\hline No & 1 & $20 \%$ \\
\hline Total & 5 & $100 \%$ \\
\hline
\end{tabular}

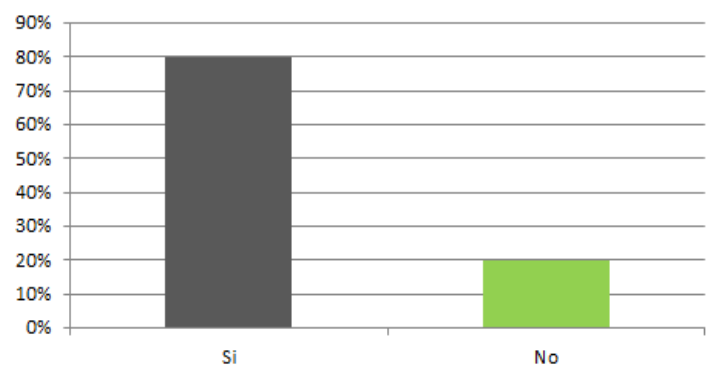

Fuente: Elaboración Propia

Gráfica 11. Gestión Ambiental

\section{¿Cuenta con una politica de gestion ambiental?}

\begin{tabular}{|l|r|r|}
\cline { 2 - 3 } \multicolumn{1}{c|}{} & Frecuencia & Porcentaje \\
\hline Si & 1 & $20 \%$ \\
\hline No & 3 & $60 \%$ \\
\hline En proceso & 1 & $20 \%$ \\
\hline Total & 5 & $100 \%$ \\
\hline
\end{tabular}

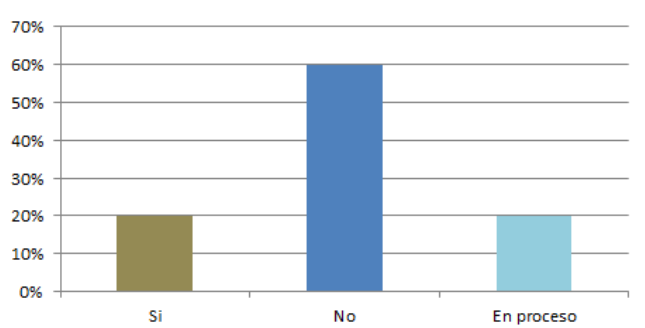


Gráfica 12. Programa 3R

¿Tiene programas de aplicación de las 3R?

\begin{tabular}{|l|r|r|}
\cline { 2 - 3 } \multicolumn{1}{c|}{} & Frecuencia & Porcentaje \\
\hline Si & 1 & 20 \\
\hline No & 2 & 40 \\
\hline En proceso & 2 & 40 \\
\hline Total & 5 & $100 \%$ \\
\hline
\end{tabular}

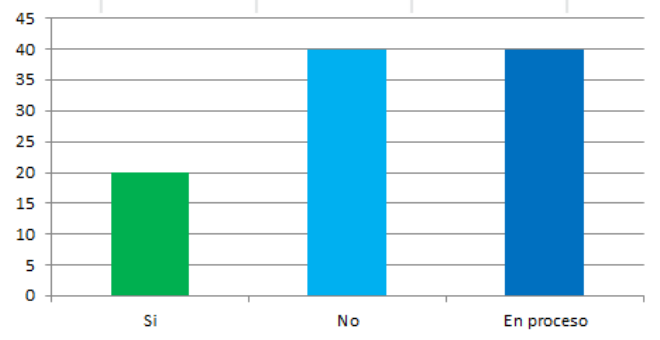

Fuente: Elaboración Propia

Gráfica 13. Proveedores

\section{Proveedores}

¿Se tiene en cuenta como proveedores a los productores locales?

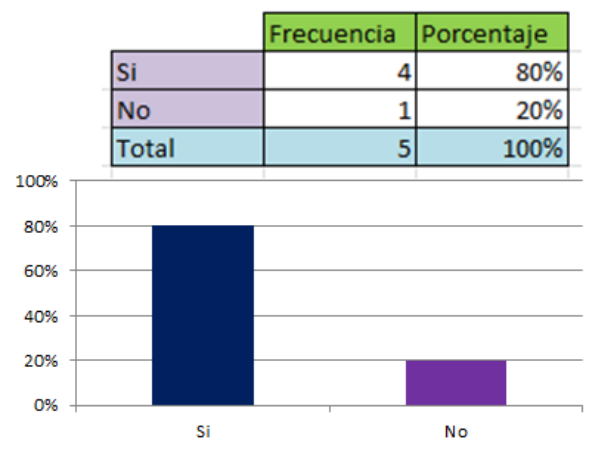

Fuente: Elaboración Propia

Gráfica 14. Mercado

\section{Mercado}

¿Tiene la empresa una política o procedimiento formal de comunicación?

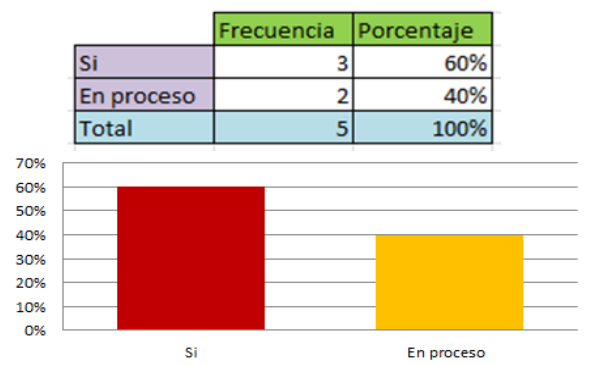

Gráfica 15. Gestión de nuevos proyectos

\section{¿Cuenta con un fondo para la gestión de nuevos proyectos?}

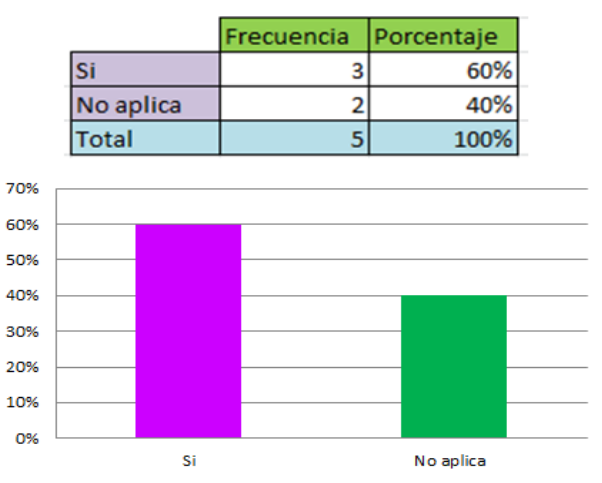

Fuente: Elaboración Propia

Gráfica 16. Comunidades

\section{Comunidades}

¿Cuenta con políticas que reducen los impactos en la comunidad inmediata tales como generación de basura, malos olores, tráfico vehicular excesivo y contaminación auditiva?

\begin{tabular}{|l|r|r|}
\cline { 2 - 3 } \multicolumn{1}{c|}{} & Frecuencia & Porcentaje \\
\hline Si & 4 & $80 \%$ \\
\hline No & 1 & $20 \%$ \\
\hline Total & 5 & $100 \%$ \\
\hline
\end{tabular}

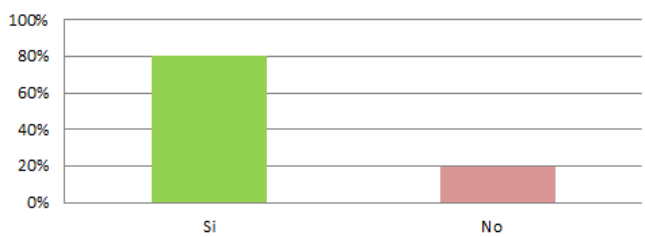

Fuente: Elaboración Propia

Gráfica 17. Organizaciones comunitarias ¿Trabaja con organizaciones comunitarias
y desarrolla proyectos conjuntamente?

\begin{tabular}{|l|r|r|}
\cline { 2 - 3 } \multicolumn{1}{c|}{} & Frecuencia & Porcentaje \\
\hline Si & 3 & $60 \%$ \\
\hline No & 2 & $40 \%$ \\
\hline Total & 5 & $100 \%$ \\
\hline
\end{tabular}

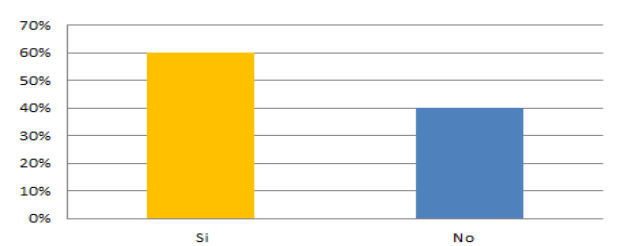


Gráfica 18. Inversión Social

¿Cuenta con un presupuesto destinado para obras de inversión social?

\begin{tabular}{|l|r|r|}
\cline { 2 - 3 } \multicolumn{1}{c|}{} & Frecuencia & Porcentaje \\
\hline Si & 1 & $20 \%$ \\
\hline No & 3 & $60 \%$ \\
\hline En proceso & 1 & $20 \%$ \\
\hline Total & 5 & $100 \%$ \\
\hline
\end{tabular}

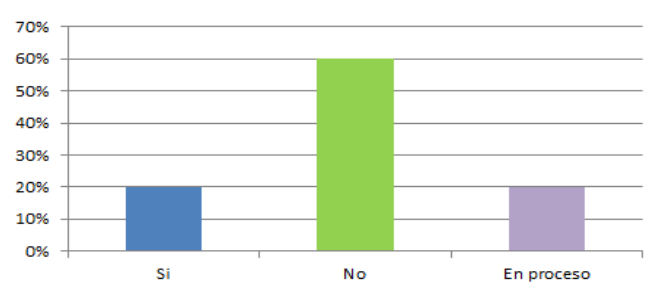

Fuente: Elaboración Propia

Gráfica 19. Proyectos Sociales

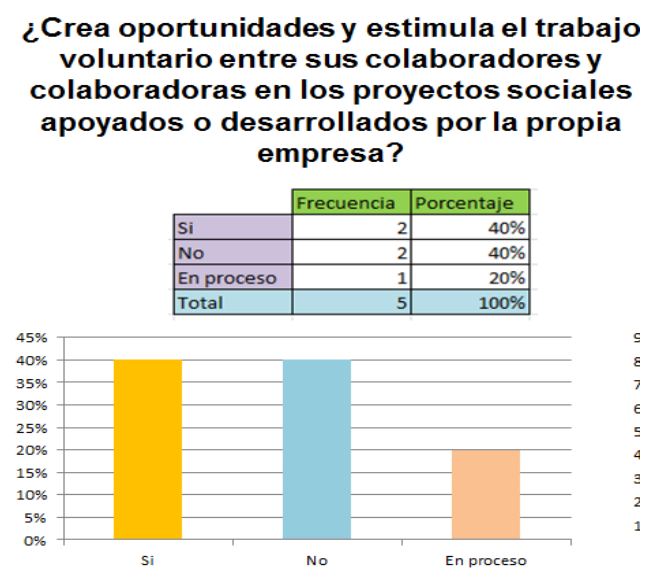

Fuente: Elaboración Propia

Gráfica 20. Colaboración

- ¿La empresa busca la asociación con otras empresas y organizaciones con el fin de compartir conocimientos y experiencias y desarrollar nuevas oportunidades de negocio?

\begin{tabular}{|l|r|r|}
\cline { 2 - 3 } \multicolumn{1}{c|}{} & Frecuencia & Porcentaje \\
\hline Si & 4 & $80 \%$ \\
\hline No & 1 & $20 \%$ \\
\hline Total & 5 & $100 \%$ \\
\hline
\end{tabular}

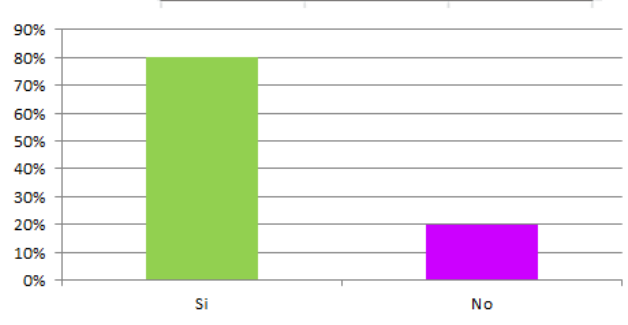

\section{DISCUSIÓN.}

El presente análisis tiene como finalidad recoger las principales incidencias que se detectaron en las empresas de la región Tula-Tepeji, con la información obtenida, se podrá establecer un modelo de RSE en el nodo Tula, del cual las empresas puedan valerse al momento de gestionar acciones de RSE y una vez que puedan madurarlas lo suficiente, poder lograr un distintivo de ESR. Las empresas participes en la investigación con relación a su gobernabilidad están constituidas legalmente, además conocen y cumplen con los requerimientos legales que aplican para el funcionamiento en sus actividades conforme a su sector, como lo muestra la gráfica 1, no obstante, se detecta que no cuentan con códigos de ética fundamentales para su desarrollo empresarial a pesar de poseer políticas referentes a la prohibición de prácticas ilegales para obtener sus ventajas competitivas.

Las empresas socias muestran desconocimiento al concepto de Responsabilidad Social Empresarial y por lo mismo en el $80 \%$ de estas, no existe área o persona a cargo de ello, no se encontró evidencia de criterios e indicadores para medir su impacto, solo en una este rubro se encuentra en proceso como lo muestra la gráfica 2.

Considerando las actualizaciones en las TIC'S solo un $40 \%$ ha adaptado estrategias en el uso del software, sistemas de información y/o canales de venta que faciliten la comunicación y gestión de información con sus grupos de interés de acuerdo con la gráfica 3. 
Una parte importante de una empresa, es su público interno, el cual debe mantenerse bajo condiciones de trabajo favorables para desarrollo dentro de la misma. Por lo cual es indispensable establecer contratos formales de trabajo donde se definan políticas y/o reglamentos para su bien común. De las empresas socias el $20 \%$ no cuenta con contratos de trabajo por escrito y otro $20 \%$ está en proceso, este factor no ha sido impedimento para que los trabajadores reciban todas las prestaciones laborales de ley ya que el $100 \%$ las ofrecen, además el $60 \%$ de estas empresas cuentan con reglamentos internos para su mejor funcionamiento como lo señala la gráfica 4, uno de los más importantes es, con respecto a higiene, salud y seguridad laboral, ya que ayudan a prevenir situaciones de riesgo o accidentes laborales.

Solo una de las empresas socias no cuenta ni tiene conocimiento del reglamento de seguridad, higiene, y medio ambiente como lo señala la gráfica 5.

Con relación a la gráfica 6 , en un $80 \%$ de las empresas socias no existe un programa formal de inducción dirigido a los nuevos integrantes de la organización, lo que pudiera significar el desconocimiento de las áreas, departamentos e inclusive de los demás colaboradores siendo un obstáculo a su desempeño laboral. Para el correcto funcionamiento de una empresa es necesario contar con personal con las cualidades $y$ capacidades que se requiere para cada área, por eso es importante llevar acabo un procedimiento formal de reclutamiento y selección personal, solo el $40 \%$ cuenta con éste, el resto está en proceso o no cuenta con él.

También es necesario darle continuidad al desarrollo tanto personal y profesional de las y los colaboradores con el objetivo de fomentar la productividad organizacional, obteniendo así que el $80 \%$ se encuentra en proceso de integrar este tipo de programas como ha quedado demostrado en las gráficas 7 y 8 respectivamente.

Para el área de medio ambiente y con relación a la gráfica 9, es fundamental que las empresas conozcan las leyes ambientales al menos nacionales que se adapten a su negocio, el $60 \%$ afirmó tener un conocimiento sobre ellas, sin en embargo es contradictorio que solo el $20 \%$ no cumple con estas leyes, las cuales les ayudarían a medir su impacto que genera en el ambiente, el 80\% sí identifica estos índices.

Conocer el impacto ambiental en algunas ocasiones, no significa que se tenga una política de gestión ambiental, como se muestra en los resultados de la gráfica 11 , donde el $60 \%$ de las empresas participantes no cuenta con evidencia al respecto.

Pudiera ser que ésta cuestión tiene como antecedente la administración o la medición de indicadores que ayuden al control y manejo de los insumos, incluso los productos y subproductos de las empresas, y que cabe mencionar que en la mayoría de los casos va de la mano con programas 
de aplicación de las 3R (reciclar, reducir y reutilizar), sin importar a que sector pertenezca la empresa. Con relación a este tema y de acuerdo a la gráfica 12 , se identificó que solo 1 de las 5 empresas participantes tiene éste tipo de programas.

Toda empresa que deseé impactar en cuanto a la calidad de sus productos o servicios, debe considerar a sus proveedores como un pilar importante dentro de sus procesos, basándose en criterios de selección tales como precio, calidad, plazo de entrega y su veracidad, es decir que esté constituido legalmente, en la gráfica 13 podemos encontrar que el $80 \%$ de estas empresas demostró que toma en cuenta a los proveedores locales, favoreciendo a la economía de la región e indicando que los productores locales cuentan con buenos estándares de calidad, por lo tanto se observó que con esto el $100 \%$ de las empresas minimiza sus costos logísticos.

La fidelidad del cliente es otro de los elementos que lleva a las empresas a generar sistemas o políticas para tener un procedimiento formal de comunicación hacia el mercado en la gráfica 14 se identificó, que con el fin de integrar esfuerzos para fortalecer sus productos o servicios, el $60 \%$ de las empresas socias, está en proceso de éste indicador y el resto tiene en claro el valor que se le debe de dar a la atención al cliente, esta variable es muy importante dentro del estudio, debido a que de esta manera se obtendrá la noción necesaria para conocer si una empresa puede satisfacer la demanda de sus clientes reales y/o potenciales.
Hoy en día ha tomado mayor trascendencia el cuidado del medio ambiente, por eso se tiene en cuenta que los productos que se ofrecen no afecten tanto a la salud de las personas como al medio ambiente.

Las empresas han tomado un papel importante para el desarrollo y apoyo tanto de la comunidad como en el ámbito estudiantil, todo esto a través de la creación de comités universidad-empresaestado, donde ambos participan para obtener un beneficio mutuo, pero en los resultados se detectó que ninguna de las empresas participa en estos.

Tener mejoras continuas o cambios en la empresa, productos y/o servicios traen consigo beneficios, en los resultados se observó que el $100 \%$ de las empresas participantes si han realizado cambios en los últimos 3 años dejando en claro que buscan la innovación.

Si se cuenta con un procedimiento que ayude a direccionar a un mejoramiento organizacional, las empresas que cuenten con éste, podrían evitar una continua rotación de personal.

Gestionar nuevos proyectos en la empresa habla bien de las mismas ya que muestra lo visionarios que son, solo el $60 \%$ de éstas, cuenta con un fondo para ejecutarlos como lo ha reflejado la gráfica 15 . Dentro de la empresa se debe de concientizar sobre los impactos en la comunidad o en las comunidades inmediatas, por lo cual deberían de existir políticas que abarquen temas como la generación de basura, malos olores, tráfico vehicular excesivo y contaminación auditiva; ésta situación se puede observar en la gráfica 16, que 
nos señala que el $80 \%$ de las empresas sí cuenta con dichas políticas, además en la gráfica 17 se señala que el $60 \%$ de éstas trabaja conjuntamente con organizaciones comunitarias para el desarrollo de nuevos proyectos. Contrastante con lo identificado en la gráfica 18 en donde el $80 \%$ no cuenta con un presupuesto destinado a obras de inversión social, además y con relación a la gráfica 19, sólo el $40 \%$ crea oportunidades y estimula el trabajo voluntario entre sus colaboradores y colaboradoras en los proyectos sociales apoyados o desarrollados por la propia empresa, además que con relación a la gráfica 20 manifiestan una voluntad abierta para colaborar y participar con otras organizaciones con el fin de crecer $y$ mantener su valor en el mercado.

Para finalizar éste apartado, vale la pena mencionar las aportaciones de autores que han realizado estudios sobre modelos de RSE, para ello y de acuerdo con Pasquero (2005), uno de los principales retos a los cuales se enfrenta actualmente la teoría de la RSE es su medición, debido a que hasta hoy, no existe un sólo método para evaluar la RSE, en este sentido Gond (2008) señala que en el mundo existen más de 227 modelos de RSE que han sido elaborados por investigadores, universidades e instituciones, principalmente. En México se cuenta con modelos como los establecidos por el Centro Mexicano para la Filantropía (Cemefi), que resulta una referencia esencial debido a que no sólo utiliza estándares locales, sino internacionales y ha sido utilizado por otros estudios para medir la RSE en México.

Si bien es cierto que el modelo Cemefi ha sido referencia para varias investigaciones, así como para el sector empresarial para otorgar el distintivo ESR, al utilizarlo de acuerdo con Villafan y Ayala (2014), se pueden encontrar dos debilidades; la primera es que existe sesgo en algunas de las preguntas, la segunda referente a su aplicación, limitada solamente a empresas, omite la opinión de los stakeholders o grupos de relación. Sin embargo, al utilizar este modelo, adaptado con el uso del proceso analítico jerárquico, es posible tener una referencia inicial o "fotografía” más precisa de la RSE.

En el presente trabajo el instrumento de recolección de información estuvo basado en los principios generales de RSE, por lo que la propuesta del modelo para el nodo Tula, es resultado del análisis de las prácticas que actualmente realizan las empresas socias de la región Tula-Tepeji y de las cuales se da evidencia en la figura 1 , en donde como se puede observar el modelo, quedo constituido por 6 variables, mismas que se deberán de difundir entre las empresas interesadas en realizar prácticas de RSE en la región, con el objetivo final de fomentar esta práctica que les permita un mejor posicionamiento con sus grupos de interés. 
Figura 1. Propuesta del Modelo de RSE nodo Tula.

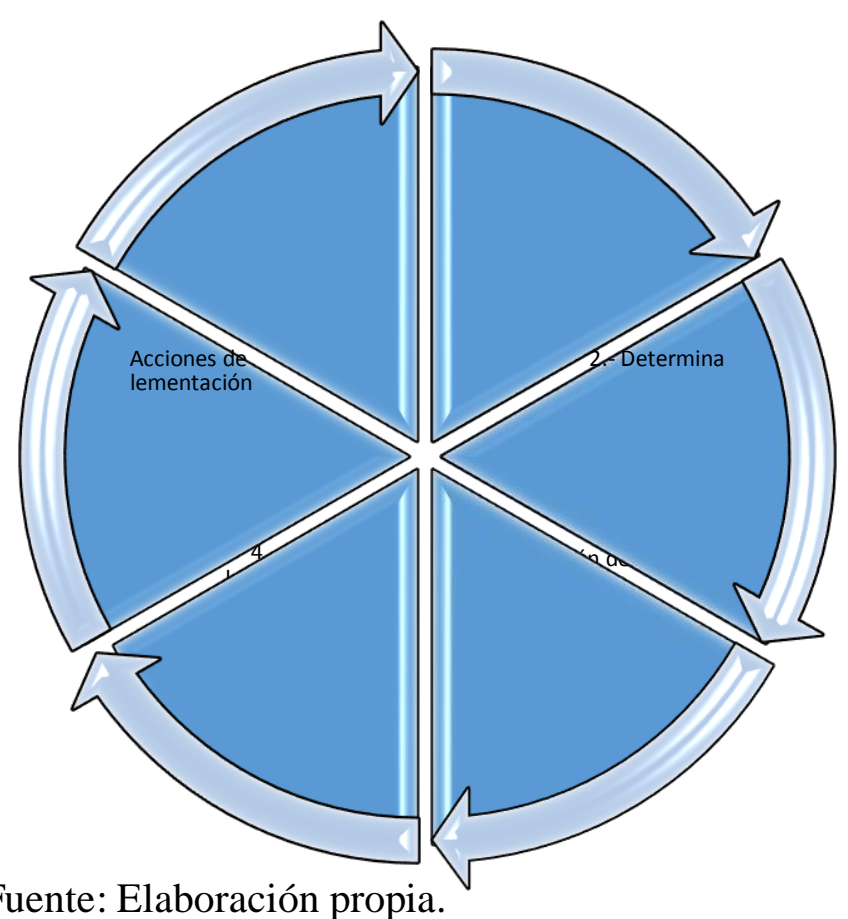

Fuente: Elaboración propia.

\section{CONCLUSIONES}

La migración hacia prácticas de RSE por parte de las organizaciones en México siguen siendo escazas, sobre todo en pequeñas y medianas empresas; éstas deben mejorar sus acciones de responsabilidad social para lograr ventajas competitivas, mismas que se puede observar no solo en sus procesos, sino también en las acciones sociales desarrolladas para con la comunidad, esto implica prácticas e interacciones con diversos públicos, entre los que se encuentran colaboradores, consumidores, clientes, proveedores, medio ambiente, gobierno y sociedad; por lo que es importante fomentar estas actividades con la finalidad de que las PYME no sólo estén mejor posicionadas, sino que además se comience a permear una cultura organizacional que permita aumentar su reputación e imagen como empleador, productor o actor de la vida local, situación que influirá en su competitividad.

Es importante enfatizar nuevamente que el presente trabajo es la primera parte del estudio longitudinal que se realiza en coordinación con la Red Iberoamericana de Responsabilidad Social Empresarial para PYME, por lo que el objetivo para éste avance el cual pretendía; integrar un modelo de RSE para las PYME de la región TulaTepeji en el Estado de Hidalgo, ha quedado de manifiesto como se muestra en la figura 1 , mismo que se derivó del análisis de las empresas socias del nodo Tula, en donde se identificaron las condiciones actuales con relación a las prácticas de RSE que las mismas realizan y que como se pudo observar son escazas, no obstante las empresas socias muestran una gran disponibilidad para implementar acciones que les permitan avanzar en este tema.

Si bien el trabajo recién comienza, ahora se tienen un punto de partida que se podrá compartir con los demás miembros de la red, quienes a su vez integrarán la propuesta de "modelo de RSE" para las PYME de Iberoamérica, esta aportación será muy enriquecedora no sólo para las PYME que estén dispuestas a establecer prácticas de responsabilidad social, sino también a las que 
decidan influir desde su posición (como PYME), mucho más que las grandes organizaciones en la salud, prosperidad y estabilidad de la comunidad en la que operan, ya que la mayor parte de sGarriga, E. y clientes y trabajadores provienen del área aledaña, por lo tanto es menester de ellas cuidar a sus grupos de interés, como lo muestra el punto 6 corond, J. P. (2008). La Responsabilité Sociale de relación a la evaluación de acciones del modelo presentado. l'entreprise. Paris: Presses Universitaires de France.

Instituto Nacional de Estadística Geografía e Informática.
"La responsabilidad social no es compromiso de unos cuantos, debemos sumar acciones y contribuir a dejar a las futuras generaciones un mundo mejor del que hemos tenido. ¿Te sumas?”
Censos Económicos 2014. Recuperado desde internet

en: proyectos/censos/ce2009/default.asp?s=est\&c=14 $\underline{220}$

\section{REFERENCIAS}

ISO (2010). ISO 26000 Project Overview. Recuperado de: Altonivel (2009). Gigantes apoyan a pequeñas empresas, Altonivel. Recuperado de ew.pdf http://www.altonivel.com.mx

Marín, L. (2007). i I need you too! Corporat identiy Bernal C. (2010) Metodología de la Investigación. Colombia. Pearson. atractiveness for consumers and the role of Social Responsibility. Journal of Buiness Ethics. Tomo Centro Mexicano para la Filantropía A.C. (2017). 71 No. 3.

Responsabilidad Social Empresarial Recuperađbercado, P. y García, P. (2007). La Responsabilidad Social En de: http://www.cemefi.org/

Espinosa, R. Contreras, R. y Rodríguez R. (2016): “Cómo puede participar las PYMES mexicanas en la RSE para aumentar su competitividad", Revista TECSISTECATL, n. 19 (abril 2016). Recuperado de:

Moreno, A. (2006). Percepción y práctica de los líderes http://www.eumed.net/rev/TECSISTECATL/n19/ competitividad.html

García. J. y H. Zabala (2008). Políticas de Estado como Empresas Del Valle De Toluca (México): Un Estudio Exploratorio. Estudios Gerenciales. Vol.23, N.102, Pp.119-135.

Recuperado de: http://www.scielo.org.co/scielo.php?script=sci artte xt\&pid=S0123-59232007000100005 de Pymes de Caracas con respecto a la Responsabilidad Social Empresarial. Trabajo de grado Msc. de la Universidad Católica Andrés sustento de la organización ciudadana. Centro de 
bello. Facultad de Ciencias Económicas y Sociales. Escuela de Ciencias Sociales.

Moreno, Z. y Graterol, D. (2001) Prácticas de responsabilidad social empresarial en la Pyme. Un estudio en el sector metalmecánico de Barquisimeto, Estado Lara, Venezuela. Contaduría y Administración, 175-194. Recuperado de:

http://www.scielo.org.mx/scielo.php?script=sci_a $\underline{\text { rttext\&pid=S0186-10422011000300009 }}$

Mota de Cabrera, C. (2006). El rol de la escritura dentro del currículo de la enseñanza y aprendizaje del inglés como segunda lengua (esl/efl): Una perspectiva histórica. Acción Pedagógica, 15(1), 56-63. Recuperado de http://www.saber. ula.ve/accionpe/ Münch, L. y Ángeles, E. (2012) Métodos y técnicas de investigación. México Trillas.

Páez. T. (2007). Teoría y práctica de la ética y la Responsabilidad Social de la Pyme Venezolana. Revista FACES Universidad de Carabobo. Volumen XVIII No. 2: 165-183.

Pasquero, J. (1995). L'environnement sociopolitique de l'Enterprise. La gestión des organisations: une approche systémique, conceptualle et stratégique. Montréal: Cheneliére/Mc Graw-Hill.

Rivero, P. (2016) La responsabilidad social corporativa en las PYMES. Lan Harremanak, I, 77-91. Recuperado de: http://www.ehu.eus/ojs/index.php/Lan_Harreman ak/article/view/3442

Saavedra, G. (2014). La PYME como generadora de empleo en México. Clío América, 8 (16), 153 172. Recuperado de: http://econpapers.repec.org/article/col000506/015 $\underline{024 . h t m}$

Villafan, K. y Ayala, D. (2014). Responsabilidad social de las empresas agrícolas y agroindustriales aguacateras de Uruapan, Michoacán, y sus implicaciones en la competitividad. Contaduría y Administración, vol.59, n.4, pp.223-251. Recuperado de: http://www.scielo.org.mx/scielo.php?script=sci_a $\underline{\text { rttext\&pid=S0186-10422014000400010 }}$ 